\title{
Sub-Ångstrom Resolution through Aberration-Corrected STEM
}

\author{
S. J. Pennycook, ${ }_{* *}^{*}$ A. R. Lupini, ${ }^{*}$ M. Varela, ${ }^{*}$ A. Borisevich, ${ }^{*}$ Y. Peng, ${ }^{*}$ M. F. Chisholm, ${ }^{*}$ N. Dellby, ${ }^{* *}$ \\ O. L. Krivanek, ${ }^{* *}$ P. D. Nellist, ${ }^{* *}$ S. Z. Szilagyi* and G. Duscher, ${ }^{* * *}$ \\ ${ }^{*}$ Condensed Matter Sciences Division, Oak Ridge National Laboratory, Oak Ridge, TN. \\ ${ }^{* *}$ Nion Co., 1102 8th St., Kirkland, WA. \\ *** Department of Materials Science and Engineering, North Carolina State University, Raleigh, NC.
}

Installation of an aberration-corrector on the VG Microscopes' HB603U STEM at Oak Ridge National Laboratory has brought immediate benefits through improved resolution, image contrast and signal to noise ratio [1]. Figure 1 shows the improvement predicted theoretically after correction of third order aberrations from an aberration limited $1.26 \AA$ probe to a $0.5 \AA$ probe. The experimental Ronchigram indeed shows the anticipated expansion of the region of flat phase by a factor of $\sim 2.5$, but the image resolution is still limited by probe instabilities. Improved contrast is seen in the line trace of Si 110 , and Fig. 2 shows contrast decreasing smoothly to $0.84 \AA$ in the power spectrum of $1 \overline{1} 00 \mathrm{SiC}$. Weak spurious spots are also present due to the probe instabilities, and it is hoped these will be cured in the near future.

More spectacular is the improved sensitivity for the imaging of single atoms. Figure 3 compares images of single La atoms on a -alumina support before (a) and after (b, d) aberration correction. Although individual atoms are visible in both images, they show substantially more contrast and improved signal to noise ratio after correction. Such images also provide an independent measure of probe size. A histogram of the full-width-half-maxima (FWHM) of intensity profiles across single La atoms (Fig. 3 (c)) gives the probe FWHM as about $0.7-0.8 \AA$. Line scans were taken in the vertical direction to avoid the effects of drift, which was predominantly in the horizontal direction. We believe the spurious measurements of large or small FWHM are due to atomic motion during the image recording, either of the specimen as a whole or of the individual atoms.

The smaller probe also provides a greatly improved image from the alumina itself, despite its very low atomic number. Although very faint lattice fringes can be discerned in the uncorrected image (arrowed), after correction, individual Al/O columns are clearly seen in the [100] zone axis image, Fig. 3 (c). Now the La atom positions are visible simultaneously with the spinel lattice of $-\mathrm{Al}_{2} \mathrm{O}_{3}$, and are seen to directly coincide with (100) atomic columns. Images such as these allow atomic location of small clusters on insulating surfaces by direct inspection. With simulations it will be possible to see and locate defect clusters within solids, determine their lattice location, and through EELS, determine their identity and chemical valence. Single atom sensitivity in EELS appears entirely feasible.

\section{References:}

[1] S. J. Pennycook et al., Proc. MRS 748 (2003) G1.1

[2] This work was supported by the USDOE under contract DE-AC05-00OR22725 managed by UTBattelle, LLC. 

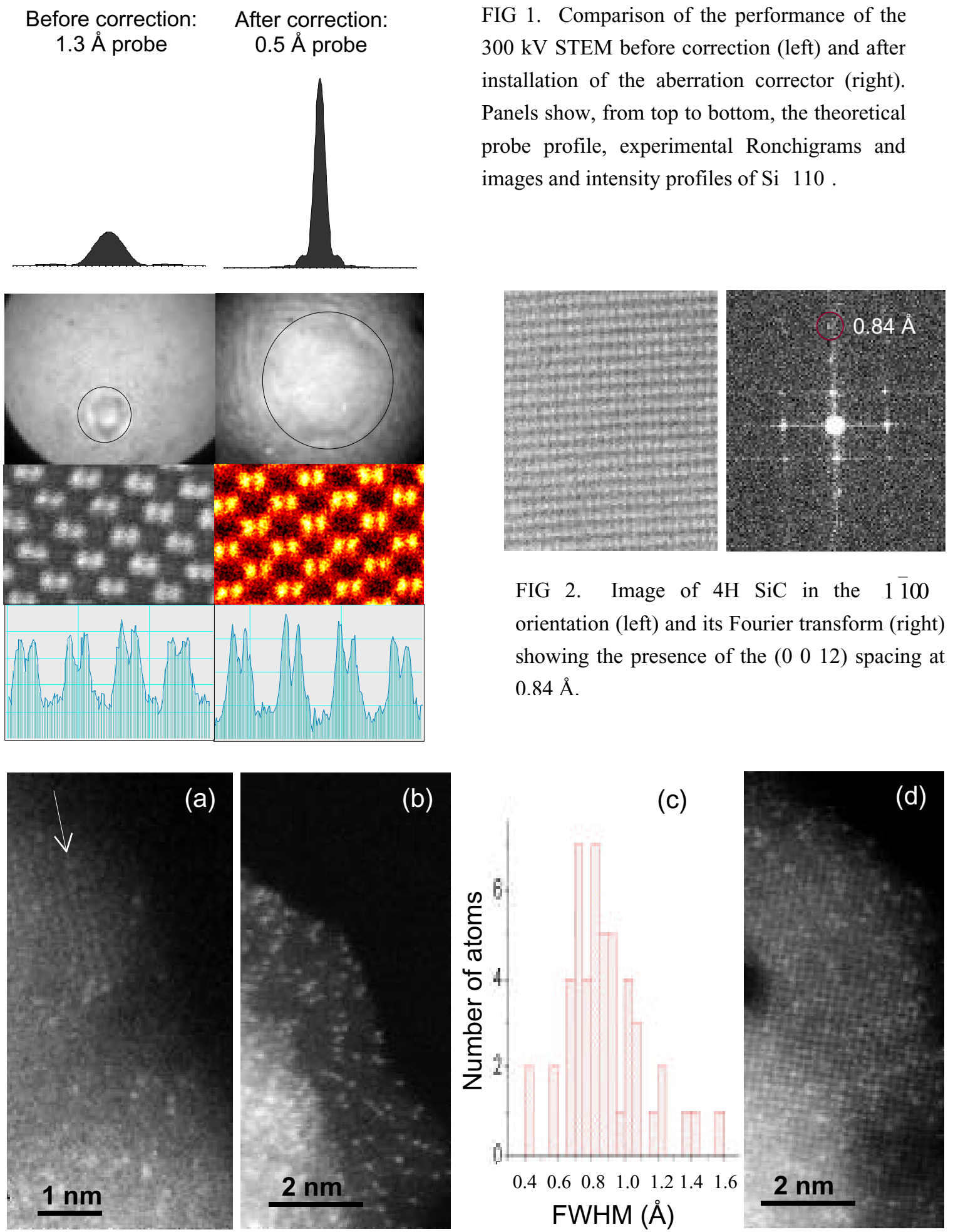

FIG 3. Images of La-doped alumina obtained with a $300 \mathrm{kV}$ STEM before (a) and after (b,d) aberration correction, showing improvement in contrast, resolution and signal to noise ratio. In (b) single La atoms are seen on a randomly oriented substrate, with (c) a histogram of FWHM of intensity profiles. In (d) La atoms on a $\mathrm{Al}_{2} \mathrm{O}_{3}$ lattice viewed in the [100] direction. 